\title{
Pressure and recurrence
}

by

\section{Véronique Maume-Deschamps (Dijon), Bernard Schmitt (Dijon), Mariusz Urbański (Denton, TX) and Anna Zdunik (Warszawa)}

\begin{abstract}
We deal with a subshift of finite type and an equilibrium state $\mu$ for a Hölder continuous function. Let $\alpha^{n}$ be the partition into cylinders of length $n$. We compute (in particular we show the existence of the limit) $\lim _{n \rightarrow \infty} n^{-1} \log \sum_{j=0}^{\tau_{n}(x)} \mu\left(\alpha^{n}\left(T^{j}(x)\right)\right)$, where $\alpha^{n}\left(T^{j}(x)\right)$ is the element of the partition containing $T^{j}(x)$ and $\tau_{n}(x)$ is the return time of the trajectory of $x$ to the cylinder $\alpha^{n}(x)$.
\end{abstract}

1. Introduction. Let $T: X \rightarrow X$ be an ergodic measure preserving transformation with respect to a probability measure $\mu$ on $X$. Let $\alpha$ be a countable measurable generating partition of $X$ with finite entropy and for every $x \in X$ let $\alpha^{n}(x)$ denote the only element of the partition $\alpha^{n}=$ $\bigvee_{j=0}^{n-1} T^{-j}(\alpha)$ containing $x$. Put

$$
\tau_{n}(x)=\min \left\{j \geq 1: T^{j}(x) \in \alpha^{n}(x)\right\} .
$$

Ornstein and Weiss proved in $[\mathrm{OW}]$ that for $\mu$-a.e. $x \in X$,

$$
\lim _{n \rightarrow \infty} \frac{1}{n} \log \tau_{n}(x)=\mathrm{h}_{\mu},
$$

where $\mathrm{h}_{\mu}$ is the measure-theoretical entropy of the mapping $T: X \rightarrow X$ with respect to the measure $\mu$.

Assume now additionally that $T: X \rightarrow X$ is a subshift of finite type, $\phi$ : $X \rightarrow X$ is a Hölder continuous potential, $\mu=\mu_{\phi}$ is the unique equilibrium state (Gibbs measure) of $T$ and $\phi$, and $\alpha$ is the partition of $X$ into initial

2000 Mathematics Subject Classification: Primary 37D35.

Key words and phrases: thermodynamic formalism, variational principle, equilibrium states.

The research of M. Urbański was supported in part by the NSF Grant DMS 0100078. The research of A. Zdunik was supported in part by the Polish KBN Grant 2 PO3A 00917. A. Zdunik wishes to thank the European Science Foundation (PRODYN) for supporting her visit to Dijon in November 2000. 
cylinders of length 1 . Let

$$
S_{n} \phi=\sum_{j=0}^{n-1} \phi \circ T^{j} .
$$

We shall use the following important property of Gibbs measures for Hölder continuous potentials (see, e.g., [Bo]): there exists a constant $C$ such that for every $x \in X$,

$$
C^{-1} \exp \left(S_{n} \phi(x)-n \mathrm{P}(\phi)\right) \leq \mu\left(\alpha^{n}(x)\right) \leq C \exp \left(S_{n} \phi(x)-n \mathrm{P}(\phi)\right),
$$

where $\mathrm{P}(\phi)$ is the topological pressure of $T$ and $\phi$,

$$
\mathrm{P}(\phi)=\lim _{n \rightarrow \infty} \frac{1}{n} \log \sum_{A \in \alpha^{n}} \exp \left(\sup \left(\left.S_{n} \phi\right|_{A}\right)\right) .
$$

Another important property is the following:

$$
\mathrm{P}(\phi)=\mathrm{h}_{\mu}+\int_{X} \phi d \mu .
$$

The following question arises. Given $x$, consider the sum

$$
\sum_{j=0}^{\tau_{n}(x)} \mu\left(\alpha^{n}\left(T^{i}(x)\right)\right)
$$

(the sum of the measures of the cylinders of the partition $\alpha^{n}$ along the trajectory of $x$ until time $\tau_{n}$ when this trajectory reaches the initial cylinder $\left.\alpha^{n}(x)\right)$. The question is whether the limit

$$
\frac{1}{n} \log \sum_{j=0}^{\tau_{n}(x)} \mu\left(\alpha^{n}\left(T^{i}(x)\right)\right)
$$

exists and what its value is. We shall give an answer for $\mu$ being a Gibbs measure for a Hölder continuous potential.

There are at least two naive ways to answer the above question. First, according to (1.2), the time $\tau_{n}$ is approximately $\exp \left(n \mathrm{~h}_{\mu}\right)$, while the measure of the typical cylinder is close to $\exp \left(-n \mathrm{~h}_{\mu}\right)$, so it seems that the limit should be zero.

On the other hand, an atom of the partition $\alpha^{n}$ should be visited by the trajectory of $x$ with a frequency close to $\mu\left(\alpha^{n}\right)$, thus the sum (1.6) should be rather close to

$$
\sum_{A \in \alpha^{n}} \mu(A) \tau_{n}(x) \mu(A)
$$

This suggests that the limit (1.7) should be equal to 


$$
\begin{aligned}
\lim _{n \rightarrow \infty}\left(\frac{1}{n} \log \tau_{n}(x)\right. & \left.+\frac{1}{n} \log \sum_{A \in \alpha^{n}} \mu(A)^{2}\right) \\
& =\lim _{n \rightarrow \infty}\left(\frac{1}{n} \log \tau_{n}(x)+\frac{1}{n} \log \int_{X} \mu\left(\alpha^{n}(x)\right) d \mu(x)\right)
\end{aligned}
$$

provided that this limit exists. It is then easily seen that the limit (1.9) really exists if $\mu$ is a Gibbs measure for a Hölder continuous potential $\phi$ for a subshift of finite type and equals $\mathrm{h}_{\mu}+\mathrm{P}(2 \phi)-2 \mathrm{P}(\phi)$.

Indeed, the first summand in (1.9) tends to $\mathrm{h}_{\mu}$ by (1.2), while the sum $\sum_{A \in \alpha^{n}} \mu(A)^{2}$ can be estimated by using the property of Gibbs measures

$$
\sum_{A \in \alpha^{n}} \mu(A)^{2} \asymp \sum_{A \in \alpha^{n}} \sup \exp \left(\left.2 S_{n} \phi\right|_{A}-2 n \mathrm{P}(\phi)\right)
$$

and we conclude that the limit in (1.7) should rather be equal to $\mathrm{h}_{\mu}+\mathrm{P}(2 \phi)-$ $2 \mathrm{P}(\phi)$, which is greater than 0 provided $\phi$ is not homologous to a constant (see Remark 1.2 below).

Of course, both "proofs" are wrong. They use some limit estimates for a given time $\tau_{n}$ and a growing number of cylinders $\alpha^{n}$. In particular, in the second "proof" we see that the time $\tau_{n}$ is certainly too short to visit all cylinders $\alpha^{n}$ even once. However, this incorrect proof leads us to a correct formula. More precisely, we have the following.

TheOREM 1.1. If $T: X \rightarrow X$ is a topologically mixing subshift of finite type, $\phi: X \rightarrow X$ is a Hölder continuous potential, $\mu=\mu_{\phi}$ is the unique equilibrium state of $T$ and $\phi$, and $\alpha$ is the partition of $X$ into initial cylinders of length 1 , then for $\mu$-a.e. $x \in X$,

$$
\begin{aligned}
& \lim _{n \rightarrow \infty} \frac{1}{n} \log \sum_{j=0}^{\tau_{n}(x)} \exp \left(S_{n} \phi \circ T^{j}(x)\right)=\mathrm{h}_{\mu}+\mathrm{P}(2 \phi)-\mathrm{P}(\phi), \\
& \lim _{n \rightarrow \infty} \frac{1}{n} \log \sum_{j=0}^{\tau_{n}(x)} \mu\left(\alpha^{n}\left(T^{j}(x)\right)\right)=\mathrm{h}_{\mu}+\mathrm{P}(2 \phi)-2 \mathrm{P}(\phi) .
\end{aligned}
$$

Notice that for $\phi=0$ the above theorem follows immediately from the result of Ornstein and Weiss. Thus it can be understood as its generalization.

REMARK 1.2. The value of the second limit in Theorem 1.1, $\mathrm{h}_{\mu}+\mathrm{P}(2 \phi)-$ $2 \mathrm{P}(\phi)$, is non-negative. Moreover, $\mathrm{h}_{\mu}+\mathrm{P}(2 \phi)-2 \mathrm{P}(\phi)=0$ iff $\phi$ is homologous to a constant, i.e. there exists a Hölder continuous function $g: X \rightarrow \mathbb{R}$ and a constant $c \in \mathbb{R}$ such that $\phi=g \circ T-g+c$.

Indeed, it is well known that (under the assumptions of Theorem 1.1) the function $t \mapsto P(t \phi)$ is convex and smooth and $\frac{d}{d t} P(t \phi)=\int_{X} \phi d \mu_{t \phi}$. Moreover, this function is strictly convex unless $\phi$ is homologous to a constant. 
Thus, using (1.5), we can write

$$
\mathrm{P}(2 \phi)=\mathrm{P}(\phi)+\int_{1}^{2} \frac{d}{d t} \mathrm{P}(t \phi) d t \geq \mathrm{P}(\phi)+\int_{X} \phi d \mu_{\phi}=\mathrm{P}(\phi)+\mathrm{P}(\phi)-\mathrm{h}_{\mu}
$$

and the inequality is strict if $\phi$ is not homologous to a constant. This gives the required inequality.

Roughly speaking, Theorem 1.1 means that the wrong "proof" above gives the correct answer because large cylinders $\alpha_{n}$ (i.e. cylinders of large measure) are visited by the trajectory of $x$ up to time $\tau_{n}(x)$ with a frequency close to the limit one (given by the Birkhoff Ergodic Theorem). It turns out that (typically) the time $\tau_{n}(x)$ is sufficiently long for the integral $\int_{X} \mu\left(\alpha^{n}(x)\right) d \mu(x)$ to be well approximated by the time average $\left(1 / \tau_{n}(x)\right) \sum_{j=0}^{\tau_{n}(x)} \mu\left(\alpha^{n}\left(T^{i}(x)\right)\right)$. The main tool in the (real) proof of Theorem 1.1 is provided by a detailed analysis of large deviations of the sums $S_{n}(\phi)$.

After writing this note we found out that questions in this spirit have been considered before for sequences of independent identically distributed random variables. It seems that this research was originated by a "new law of large numbers" (see [ER]), where the average $k^{-1} U_{n}, U_{n}=\max _{0 \leq i \leq n-k}\left(X_{i}+\right.$ $\left.\ldots+X_{i+k}\right), k=[c \log n]$, was considered. See also [DDL].

A result analogous to Theorem 1.1 in the context of independent equally distributed random variables appeared in [To].

2. Proofs. Let $T: X \rightarrow X$ be an ergodic measure preserving transformation with respect to a probability measure $\mu$ on $X$. Let $\phi$ be a bounded measurable function defined on $X$.

We introduce the following notation.

$$
c_{\phi, \mu}(t)=\limsup _{n \rightarrow \infty} \frac{1}{n} \log \int \exp \left(S_{n}(t \phi)\right) d \mu
$$

where $S_{n} \phi$ is defined in (1.3). In statistical mechanics, $c_{\phi, \mu}(t)$ is usually called the free energy function, provided that limsup can be replaced by lim. The notion of free energy is closely related to the notion of topological pressure. We have the following simple

Lemma 2.1. If $T: X \rightarrow X$ is a topologically mixing subshift of finite type, $\phi: X \rightarrow X$ is a Hölder continuous potential and $\mu_{\phi}$ is the unique equilibrium state of $T$ and $\phi$, then $c_{\phi, \mu_{\phi}}(t)=\mathrm{P}((t+1) \phi)-\mathrm{P}(\phi)$, where $\mathrm{P}((t+1) \phi)$ is the topological pressure of the function $(t+1) \phi$. In particular, in this case, limsup can be replaced by $\lim$ in the definition of $c_{\phi, \mu_{\phi}}$. 
Proof. As before, denote by $\alpha$ the partition into cylinders of length 1 . It is well known that for every $x \in X$,

$$
\mu_{\phi}\left(\alpha^{n}(x)\right) \asymp \exp \left(S_{n} \phi(x)-\mathrm{P}(\phi) n\right)
$$

(see (1.4)), where $A \asymp B$ means that the quotients $A / B$ and $B / A$ are uniformly bounded from above (and so also from below). For every cylinder $A \in \alpha^{n}$ choose one point $x_{A} \in A$. We then get

$$
\begin{aligned}
\log \int \exp \left(S_{n} t \phi\right) d \mu & { }_{+} \log \sum_{A \in \alpha^{n}} \mu_{\phi}(A) \exp \left(S_{n} t \phi\left(x_{A}\right)\right) \\
& \asymp+\log \sum_{A \in \alpha^{n}} \exp \left(S_{n} \phi\left(x_{A}\right)-\mathrm{P}(\phi) n\right) \exp \left(S_{n} t \phi\left(x_{A}\right)\right) \\
& =\log \sum_{A \in \alpha^{n}} \exp \left(S_{n}((t+1) \phi)\left(x_{A}\right)-\mathrm{P}(\phi) n\right),
\end{aligned}
$$

where $A \asymp_{+} B$ means that the differences $A-B$ and $B-A$ are uniformly bounded from above (and so also from below). It now immediately follows from the definition of $c_{\phi, \mu_{\phi}}(t)$ and from the definition of topological pressure that

$$
c_{\phi, \mu_{\phi}}(t)=\mathrm{P}((t+1) \phi)-\mathrm{P}(\phi)
$$

REMARK 2.2. We use both free energy and pressure even though these notions are very closely related to each other in our case. The free energy is usually used in the statement of the Large Deviation Theorem, which will be our main tool in the proof of Lemma 2.4.

To prove Theorem 1.1, we shall estimate the $\operatorname{sum} \sum_{j=0}^{\tau_{n}(x)} \exp \left(S_{n} \phi \circ T^{j}(x)\right)$ from above (Proposition 2.3) and from below (Lemma 2.4). Notice that the estimate from above holds under much weaker assumptions than the estimate from below.

Proposition 2.3. Suppose that $T: X \rightarrow X$ is an ergodic measure preserving transformation with respect to a probability measure $\mu$, and the partition $\alpha$ and the time $\tau_{n}(x)$ are defined as in (1.1). Let $\phi$ be a bounded measurable function. Then for $\mu$-a.e. $x \in X$,

$$
\limsup _{n \rightarrow \infty} \frac{1}{n} \log \sum_{j=0}^{\tau_{n}(x)} \exp \left(S_{n} \phi \circ T^{j}(x)\right) \leq \mathrm{h}_{\mu}+c_{\phi, \mu}(1) .
$$

Proof. Put

$$
g_{n}(x)=\exp \left(S_{n} \phi(x)\right)
$$

Fix $\varepsilon>0$ and for every $n \geq 1$ consider the set

$$
B_{n}(\varepsilon)=\left\{x \in X: \sum_{j=0}^{\exp \left(\left(\mathrm{h}_{\mu}+\varepsilon / 3\right) n\right)} g_{n} \circ T^{j}(x)>\exp \left(\left(\mathrm{h}_{\mu}+c_{\phi, \mu}(1)+\varepsilon\right) n\right)\right\} .
$$


Applying Chebyshev's inequality we obtain

$$
\begin{aligned}
\mu\left(B_{n}(\varepsilon)\right) & \leq \exp \left(-\left(\mathrm{h}_{\mu}+c_{\phi, \mu}(1)+\varepsilon\right) n\right) \int \sum_{j=0}^{\exp \left(\left(\mathrm{h}_{\mu}+\varepsilon / 3\right) n\right)} g_{n} \circ T^{j} d \mu \\
& \leq \exp \left(-\left(\mathrm{h}_{\mu}+c_{\phi, \mu}(1)+\varepsilon\right) n\right) \exp \left(\left(\mathrm{h}_{\mu}+\varepsilon / 3\right) n\right) \int g_{n} d \mu \\
& =\exp \left(-\left(c_{\phi, \mu}(1)+2 \varepsilon / 3\right) n\right) \int g_{n} d \mu .
\end{aligned}
$$

But it follows from the definition of $c_{\phi, \mu}(1)$ that for all $n$ large enough, say $n \geq n_{\varepsilon}, \int g_{n} d \mu \leq \exp \left(\left(c_{\phi, \mu}(1)+\varepsilon / 3\right) n\right)$. Consequently,

$$
\mu\left(B_{n}(\varepsilon)\right) \leq \exp (-\varepsilon n / 3)
$$

for all $n \geq n_{\varepsilon}$. Thus the series $\sum_{n=1}^{\infty} \mu\left(B_{n}(\varepsilon)\right)$ converges and it follows from the Borel-Cantelli lemma that there exists a measurable set $A_{\varepsilon}^{\prime}$ such that $\mu\left(A_{\varepsilon}^{\prime}\right)=1$ and each point of $A_{\varepsilon}^{\prime}$ belongs to finitely many sets $B_{n}(\varepsilon)$ only. In particular

$$
\limsup _{n \rightarrow \infty} \frac{1}{n} \log \sum_{j=0}^{\exp \left(\left(\mathrm{h}_{\mu}+\varepsilon / 3\right) n\right)} g_{n} \circ T^{j}(x) \leq \mathrm{h}_{\mu}+c_{\phi, \mu}(1)+\varepsilon
$$

for all $x \in A_{e}^{\prime}$. Since by (1.2), $\lim _{n \rightarrow \infty} n^{-1} \log \tau_{n}(x)=\mathrm{h}_{\mu}$ for $\mu$-a.e. $x \in X$, we conclude that

$$
\limsup _{n \rightarrow \infty} \frac{1}{n} \log \sum_{j=0}^{\tau_{n}(x)} \exp \left(S_{n} \phi \circ T^{j}(x)\right) \leq \mathrm{h}_{\mu}+c_{\phi, \mu}(1)+\varepsilon
$$

for all points $x$ in some measurable set $A_{\varepsilon}$ with $\mu\left(A_{\varepsilon}\right)=1$. Putting $A=$ $\bigcap_{k \geq 1} A_{1 / k}$, we therefore have $\mu(A)=1$ and

$$
\limsup _{n \rightarrow \infty} \frac{1}{n} \log \sum_{j=0}^{\tau_{n}(x)} \exp \left(S_{n} \phi \circ T^{j}(x)\right) \leq \mathrm{h}_{\mu}+c_{\phi, \mu}(1) \quad \text { for all } x \in A .
$$

Our main technical result is the following.

Lemma 2.4. If $T: X \rightarrow X$ is a topologically mixing subshift of finite type, $\phi: X \rightarrow X$ is a Hölder continuous potential and $\mu=\mu_{\phi}$ is the unique equilibrium state of $T$ and $\phi$, then for $\mu$-a.e. $x \in X$,

$$
\liminf _{n \rightarrow \infty} \frac{1}{n} \log \sum_{j=0}^{\tau_{n}(x)} \exp \left(S_{n} \phi \circ T^{j}(x)\right) \geq \mathrm{h}_{\mu}+c_{\phi, \mu}(1) .
$$

Proof. Replacing $\phi$ by $\phi-\mathrm{P}(\phi)$ if necessary, we may assume without loss of generality that $\mathrm{P}(\phi)=0$. Now, we can also assume that $\phi<0$ in $X$. Indeed, since $\mathrm{P}(\phi)=0$, it follows that there exists $k \in \mathbb{N}$ such that for every $x$ we have $S_{k}(\phi)<0$. So, we can replace $\phi$ by $\phi^{\prime}=\left(S_{k} \phi\right) / k$. The 
Gibbs states $\mu_{\phi}$ and $\mu_{\phi^{\prime}}$ are the same. Since $\exp \left(S_{n} \phi \circ T^{j}(x)\right)$ differs from $\exp \left(S_{n} \phi^{\prime} \circ T^{j}(x)\right)$ by a bounded factor, the left-hand side of (2.1) does not change when $\phi$ is replaced by $\phi^{\prime}$. By the same reason, the right-hand side does not change either.

From now on we assume that $\mathrm{P}(\phi)=0$ and $\phi<0$ in $X$.

Assume that $\phi$ is homologous to a constant. In this case the pressure function $t \mapsto P(t \phi)$ is affine and (see Remark 1.2) $\mathrm{h}_{\mu}+P(2 \phi)-2 P(\phi)=0$. Moreover, in this case $\mu=\mu_{\phi}$ is simply the measure of maximal entropy and $\mu\left(\alpha^{n}\right) \asymp \exp \left(-n \mathrm{~h}_{\mu}\right)$. Thus, in this case the statement of Theorem 1.1 follows directly from the result of $[\mathrm{OW}]$.

So from now on, we assume that $\phi$ is not homologous to a constant. Put $\psi=\phi+\mathrm{h}_{\mu}$. We then have

$$
\begin{aligned}
\sum_{i=0}^{\tau_{n}(x)} \exp \left(S_{n} \phi\left(T^{i}(x)\right)\right) & =\exp \left(-n \mathrm{~h}_{\mu}\right) \sum_{i=0}^{\tau_{n}(x)} \exp \left(S_{n} \phi\left(T^{i} x\right)+n \mathrm{~h}_{\mu}\right) \\
& =\exp \left(-n \mathrm{~h}_{\mu}\right) \sum_{i=0}^{\tau_{n}(x)} \exp \left(S_{n} \psi\left(T^{i}(x)\right)\right) .
\end{aligned}
$$

Fix $\delta>0$. Then, using (2.2), we get

$$
\sum_{i=0}^{\tau_{n}} \exp \left(S_{n} \phi\left(T^{i}(x)\right)\right) \geq \exp \left(-n \mathrm{~h}_{\mu}\right) \exp (n \delta) \cdot \#\left\{i \in\left(0, \tau_{n}\right): S_{n} \psi(x)>n \delta\right\} .
$$

Since $\mathrm{P}(\phi)=0$, we have $\int \psi d \mu=0$. Thus the Large Deviation Theorem (see [El, Th. II.6.1]) gives

$$
\lim _{n \rightarrow \infty} \frac{1}{n} \log \mu\left(\left\{x: \frac{S_{n} \psi(x)}{n}>\delta\right\}\right)=-\widehat{I}(\delta),
$$

where $\widehat{I}(\delta)$ is the Legendre-Fenchel transform of the free energy function

$$
c_{\psi, \mu}(t)=\lim _{n \rightarrow \infty} \frac{1}{n} \log \int \exp \left(S_{n}(t \psi)\right) d \mu .
$$

Notice that $\widehat{I}(\delta)=I\left(-\mathrm{h}_{\mu}+\delta\right)$, where $I$ is the Legendre-Fenchel transform of the free energy function

$$
c_{\phi, \mu}(t)=\lim _{n \rightarrow \infty} \frac{1}{n} \log \int \exp \left(S_{n}(t \phi)\right) d \mu .
$$

For every $n \geq 1$ put

$$
B_{\delta}(n)=\left\{x: \text { there exists } y \in \alpha^{n}(x) \text { such that } \frac{S_{n} \psi(y)}{n}>\delta\right\} .
$$

Since $\psi$ is a Hölder continuous function, there exists a constant $C$ independent of $n$ such that if $y \in \alpha^{n}(x)$ then $\left|S_{n} \psi(x)-S_{n} \psi(y)\right|<C$. Fix $\varepsilon>0$. 
Since the transform $\widehat{I}$ is continuous, it follows from (2.3) that for all $n$ large enough,

$$
\mu\left(B_{\delta}(n)\right) \geq \mu\left(\left\{x: \frac{S_{n} \psi(x)}{n}>\delta-\frac{C}{n}\right\}\right)>\exp (-n(\widehat{I}(\delta)+\varepsilon)) .
$$

The idea of the computation below is the following. For an integer $M=$ $M(n)$ we shall estimate from below the number of points in the trajectory of $x$ under $T^{n}: x, T^{n}(x), \ldots, T^{M n}(x)$ which fall into the set $B_{\delta}(n)$. As a tool, we use Chebyshev's inequality together with weak dependence of the random variables $\chi_{B_{\delta}(n)} \circ T^{n j}$. More precisely, we conclude that the frequency of "times" $k \in\{1, \ldots, M\}$ such that $T^{n k}(x) \in B_{\delta}(n)$ is close to the measure of $B_{\delta}(n)$. This estimate works for all $x$ outside some set $A_{n}$, where $\mu\left(A_{n}\right)$ is close to 0 (see $(2.7),(2.8),(2.10)$ ). The "time" $M$ under consideration depends on $n$ and is related to the typical return time $\tau_{n}$ (see (2.8)). If $x$ is chosen so that for every $n>n_{0}=n_{0}(x)$ the point $x$ is not in $A_{n}$, we will get the estimate of

$$
\liminf _{n \rightarrow \infty} \frac{1}{n} \log \sum_{i=0}^{\tau_{n}(x)} \exp \left(S_{n} \phi\left(T^{i}(x)\right)\right)
$$

from below in terms of the value of Legendre-Fenchel transform of the free energy function evaluated at $\delta$ (see (2.13)). Finally, we examine the range of possible $\delta$ 's. This will lead us (using the Legendre-Fenchel transform again) to the inequality $(2.1)$.

So, let $Y_{n}=\chi_{B_{\delta}(n)}$, the characteristic function of the set $B_{\delta}(n)$. Notice that the function $Y_{n}$ is constant on each cylinder of the $n$th generation. We have $E\left(Y_{n}\right)=\mu\left(B_{\delta}(n)\right)$ and $D^{2}\left(Y_{n}\right)=\mu\left(B_{\delta}(n)\right)\left(1-\mu\left(B_{\delta}(n)\right)\right)$. For every integer $M \geq 1$ set

$$
L_{M, n}=Y_{n}+Y_{n} \circ T^{n}+\ldots+Y_{n} \circ T^{M n} .
$$

Our aim now is to estimate the variance $D^{2}\left(L_{M, n}\right)$ from above. Let $\widetilde{Y}_{n}=$ $Y_{n}-E\left(Y_{n}\right)$. Then

$$
\begin{aligned}
D^{2}\left(L_{M, n}\right)= & E\left(\left(\widetilde{Y}_{n}+\widetilde{Y}_{n} \circ T^{n}+\ldots+\widetilde{Y}_{n} \circ T^{M n}\right)^{2}\right) \\
= & (M+1) E\left(\widetilde{Y}_{n}^{2}\right)+2\left(M E\left(\widetilde{Y}_{n}\left(\widetilde{Y}_{n} \circ T^{n}\right)\right)\right. \\
& \left.+(M-1) E\left(\widetilde{Y}_{n}\left(\widetilde{Y}_{n} \circ T^{2 n}\right)\right)+\ldots+E\left(\widetilde{Y}_{n}\left(\widetilde{Y}_{n} \circ T^{M n}\right)\right)\right) .
\end{aligned}
$$

For every $l \geq 0$ let

$$
\gamma(l)=\sup \left\{\frac{\left|\mu\left(C_{n} \cap T^{-(l+n)}\left(C_{n}^{\prime}\right)\right)-\mu\left(C_{n}\right) \mu\left(C_{n}^{\prime}\right)\right|}{\mu\left(C_{n}\right) \mu\left(C_{n}^{\prime}\right)}: n \geq 1, C_{n}, C_{n}^{\prime} \in \alpha^{n}\right\} .
$$

Since the random variable $\widetilde{Y}_{n}$ is constant on each cylinder of length $n$, we get 


$$
\begin{aligned}
E\left(\tilde{Y}_{n}\left(\tilde{Y}_{n} \circ T^{j n}\right)\right) & \\
= & \left.\left.\sum_{C_{n}} \sum_{C_{n}^{\prime}} \mu\left(C_{n} \cap T^{-j n}\left(C_{n}^{\prime}\right)\right) \widetilde{Y}_{n}\right|_{C_{n}} \widetilde{Y}_{n}\right|_{C_{n}^{\prime}} \\
= & \left.\left.\sum_{C_{n}} \sum_{C_{n}^{\prime}} \mu\left(C_{n}\right) \mu\left(C_{n}^{\prime}\right) \widetilde{Y}_{n}\right|_{C_{n}} \widetilde{Y}_{n}\right|_{C_{n}^{\prime}} \frac{\mu\left(C_{n} \cap T^{-j n}\left(C_{n}^{\prime}\right)\right)}{\mu\left(C_{n}\right) \mu\left(C_{n}^{\prime}\right)} \\
= & \left.\left.\sum_{C_{n}} \sum_{C_{n}^{\prime}} \mu\left(C_{n}\right) \mu\left(C_{n}^{\prime}\right) \widetilde{Y}_{n}\right|_{C_{n}} \widetilde{Y}_{n}\right|_{C_{n}^{\prime}} \\
& +\left.\left.\sum_{C_{n}} \sum_{C_{n}^{\prime}} \mu\left(C_{n}\right) \mu\left(C_{n}^{\prime}\right) \widetilde{Y}_{n}\right|_{C_{n}} \widetilde{Y}_{n}\right|_{C_{n}^{\prime}} \frac{\mu\left(C_{n} \cap T^{-n j}\left(C_{n}^{\prime}\right)\right)-\mu\left(C_{n}\right) \mu\left(C_{n}^{\prime}\right)}{\mu\left(C_{n}\right) \mu\left(C_{n}^{\prime}\right)} .
\end{aligned}
$$

The first summand on the right-hand side is equal to $\left(E\left(\widetilde{Y}_{n}\right)\right)^{2}=0$. The second summand can be estimated from above by $\gamma((j-1) n)\left(E\left(\left|\widetilde{Y}_{n}\right|\right)\right)^{2} \leq$ $\gamma((j-1) n) E\left(\widetilde{Y}_{n}^{2}\right)$. The sequence $\{\gamma(k)\}_{k=0}^{\infty}$ converges to 0 exponentially fast (this is a well-known property of Gibbs measures, see e.g. [Bo]). Using (2.5) we obtain

$$
D^{2}\left(L_{M, n}\right) \leq(M+1) E\left(\widetilde{Y}_{n}^{2}\right)\left(1+2 \sum_{j=1}^{\infty} \gamma((j-1) n)\right) \leq C_{1} M E\left(\widetilde{Y}_{n}^{2}\right)
$$

where $C_{1}$ is some universal constant independent of $M$ and $n$. By Chebyshev's inequality we get

$$
\mu\left(\left\{x:\left|\frac{L_{M, n}(x)}{M}-\mu\left(B_{\delta}(n)\right)\right|>\eta\right\}\right)<\frac{D^{2}\left(L_{M, n} / M\right)}{\eta^{2}} .
$$

Put

$$
M=M(n)=\left[\frac{\exp \left(n\left(\mathrm{~h}_{\mu}-\varepsilon\right)\right)}{n}\right], \quad \eta=\frac{1}{2} \mu\left(B_{\delta}(n)\right)
$$

and set

$$
A_{n}=\left\{x:\left|\frac{L_{M(n), n}(x)}{M(n)}-\mu\left(B_{\delta}(n)\right)\right|>\frac{1}{2} \mu\left(B_{\delta}(n)\right)\right\} .
$$

Since $E\left(\widetilde{Y}_{n}^{2}\right)=D^{2}\left(Y_{n}\right)=\mu\left(B_{\delta}(n)\right)\left(1-\mu\left(B_{\delta}(n)\right)\right)$, using (2.4) and (2.6), for all $n$ large enough, we get

$$
\begin{aligned}
\mu\left(A_{n}\right) & \leq \frac{D^{2}\left(\frac{L_{M(n), n}}{M(n)}\right)}{\left(\frac{1}{2} \mu\left(B_{\delta}(n)\right)\right)^{2}} \leq \frac{C_{2} M(n) \mu\left(B_{\delta}(n)\right)\left(1-\mu\left(B_{\delta}(n)\right)\right)}{M(n)^{2}\left(\mu\left(B_{\delta}(n)\right)\right)^{2}} \\
& \leq C_{3} \frac{n}{\exp \left(n\left(\mathrm{~h}_{\mu}-\varepsilon\right)\right) \exp (-n(\widehat{I}(\delta)+\varepsilon))} \\
& =C_{3} n \exp \left(n\left(\widehat{I}(\delta)-\mathrm{h}_{\mu}+2 \varepsilon\right)\right),
\end{aligned}
$$


where $C_{2}$ and $C_{3}$ are some universal constants. We therefore conclude that if $\widehat{I}(\delta)<\mathrm{h}_{\mu}$ and $\varepsilon>0$ is small enough, then the series $\sum \mu\left(A_{n}\right)$ converges. Hence, by the Borel-Cantelli Lemma, for $\mu$-a.e. $x$ there exists $n_{0}=n_{0}(x)$ such that $x \notin A_{n}$ for all $n \geq n_{0}(x)$. In view of (1.2) we may assume without loss of generality that $\tau_{n}(x)>\exp \left(n\left(\mathrm{~h}_{\mu}-\varepsilon\right)\right)$ for all $n \geq n_{0}(x)$. Thus, for all $n \geq n_{0}$ we get

$$
\begin{aligned}
\#\{i \in\{0, \ldots, & \left.\left.\tau_{n}(x)\right\}: S_{n} \psi\left(T^{i}(x)\right)>n \delta\right\} \\
& \geq \#\left\{i \in\left\{0, \ldots, n^{-1} \exp \left(n\left(\mathrm{~h}_{\mu}-\varepsilon\right)\right)\right\}: T^{i}(x) \in B_{\delta}(n)\right\} \\
& \geq \frac{1}{2} \mu\left(B_{\delta}(n)\right) \exp \left(n\left(\mathrm{~h}_{\mu}-\varepsilon\right)\right) .
\end{aligned}
$$

Finally, using (2.2) and (2.4) we obtain

$$
\begin{aligned}
& \sum_{i=0}^{\tau_{n}(x)} \exp \left(S_{n} \phi\left(T^{i}(x)\right)\right) \\
& \quad \geq \frac{1}{2} \exp \left(-n \mathrm{~h}_{\mu}\right) \exp (n \delta) \exp (-n(\widehat{I}(\delta)+\varepsilon)) \exp \left(n\left(\mathrm{~h}_{\mu}-\varepsilon\right)\right) .
\end{aligned}
$$

Therefore,

$$
\liminf _{n \rightarrow \infty} \frac{1}{n} \log \sum_{i=0}^{\tau_{n}(x)} \exp \left(S_{n} \phi\left(T^{i}(x)\right)\right) \geq-\mathrm{h}_{\mu}+\delta-\widehat{I}(\delta)-2 \varepsilon+\mathrm{h}_{\mu} .
$$

Letting $\varepsilon \searrow 0$, we get

$$
\liminf _{n \rightarrow \infty} \frac{1}{n} \log \sum_{i=0}^{\tau_{n}(x)} \exp \left(S_{n} \phi\left(T^{i}(x)\right)\right) \geq \delta-\widehat{I}(\delta) .
$$

The reasoning above works for every $\delta>0$ such that the series $\sum \mu\left(A_{n}\right)$ is convergent. As we have noticed, a sufficient condition for this is that $\widehat{I}(\delta)$ $<\mathrm{h}_{\mu}$. So, in particular, one can take an arbitrary $\delta$ such that $\widehat{I}(\delta)<\delta<\mathrm{h}_{\mu}$. Notice that the domain of $I$ (the Legendre-Fenchel transform of $c_{\phi, \mu}(t)$ ) is contained in $(-\infty, 0)$ (roughly speaking, $z$ is in the domain of $I$ if there exists $t$ such that $\left.c_{\phi, \mu}^{\prime}(t)=z\right)$. Consequently, the domain of $\widehat{I}$ is contained in $\left(-\infty, \mathrm{h}_{\mu}\right)$. Therefore, the estimate $(2.13)$ is satisfied for all $\delta$ in the domain of $\widehat{I}(\delta)$ for which $\widehat{I}(\delta)<\delta$. We now argue that such $\delta$ s exist. Indeed, $\widehat{I}$ is differentiable ([El, Th. VI.5.6]) and it attains its minimum $\widehat{I}(0)=0$ (so also $\left.\widehat{I}^{\prime}(0)=0\right)$. Therefore, we have the estimate

$$
\liminf _{n \rightarrow \infty} \frac{1}{n} \log \sum_{i=0}^{\tau_{n}(x)} \exp \left(S_{n} \phi\left(T^{i}(x)\right)\right) \geq \sup _{\delta}\{\delta-\widehat{I}(\delta)\},
$$

where the supremum is over all $\delta$ in the domain of $\widehat{I}$. But, again by definition of Legendre transform, this supremum is precisely the value of the LegendreFenchel transform of $\widehat{I}$ at the point 1 . The Legendre-Fenchel transform of $I$ 
is again $c_{\phi, \mu}(t)$ (see e.g. [El, Th. VI.5.3]) and the Legendre-Fenchel transform of $\widehat{I}$ evaluated at $t$ is

$$
c_{\phi, \mu}(t)+\mathrm{h}_{\mu} \cdot t .
$$

So, its value at 1 equals $c_{\phi, \mu}(1)+\mathrm{h}_{\mu}$. This shows that

$$
\liminf _{n \rightarrow \infty} \frac{1}{n} \log \sum_{i=0}^{\tau_{n}(x)} \exp \left(S_{n} \phi\left(T^{i}(x)\right)\right) \geq \mathrm{h}_{\mu}+c_{\phi, \mu}(1) .
$$

We now get the main result of this paper, Theorem 1.1, as an immediate consequence of Proposition 2.3, Lemma 2.4 and Lemma 2.1.

The following remark along with (1.2) shows that Theorem 1.1 can be used to calculate topological pressure provided that we are given generic points of equilibrium states of the Hölder continuous potentials $2^{-j} \phi, j \geq 0$.

REMARK 2.5. If $T: X \rightarrow X$ is a continuous map of a compact metric space $X$ and if $\phi: X \rightarrow X$ is a continuous potential, then

$$
\mathrm{P}(\phi)=\mathrm{h}_{\text {top }}(T)+\sum_{j=0}^{\infty}\left(\mathrm{P}\left(2^{-j} \phi\right)-\mathrm{P}\left(2^{-(j+1)} \phi\right)\right) .
$$

Moreover

$$
\left|\mathrm{P}(\phi)-\left(\mathrm{h}_{\text {top }}(T)+\sum_{j=0}^{n}\left(\mathrm{P}\left(2^{-j} \phi\right)-\mathrm{P}\left(2^{-(j+1)} \phi\right)\right)\right)\right| \leq 2^{-(n+1)}\|\phi\|_{\infty} .
$$

Indeed, for every $n \geq 0$ we have

$$
\begin{aligned}
\mathrm{P}(\phi)=\sum_{j=0}^{n}\left(\mathrm{P}\left(2^{-j} \phi\right)-\mathrm{P}\left(2^{-(j+1)} \phi\right)\right) & +\mathrm{P}\left(2^{-(n+1)} \phi\right) \\
\left|\mathrm{P}(\phi)-\left(\mathrm{h}_{\text {top }}(T)+\sum_{j=0}^{n}\left(\mathrm{P}\left(2^{-j} \phi\right)-\mathrm{P}\left(2^{-(j+1)} \phi\right)\right)\right)\right| & =\left|\mathrm{P}\left(2^{-(n+1)} \phi\right)-\mathrm{h}_{\text {top }}(T)\right| \\
& =\left|\mathrm{P}\left(2^{-(n+1)} \phi\right)-\mathrm{P}(0)\right| \\
& \leq 2^{-(n+1)}\|\phi\|_{\infty} .
\end{aligned}
$$

Finally, let us discuss what can be proved in a more general context. In general, without the strong assumption of Theorem 1.1 we are only able to prove the following straightforward lower bound:

Proposition 2.6. Suppose that $T: X \rightarrow X$ is a transformation preserving an ergodic probability measure $\mu$. Let $\alpha$ be a countable measurable generating partition of $X$ with finite entropy and let $\tau_{n}$ be defined as in (1.1). 
Let $\phi: X \rightarrow \mathbb{R}$ be a bounded measurable function. Then for $\mu$-almost every $x \in X$ we have

$$
\liminf _{n \rightarrow \infty} \frac{1}{n} \log \sum_{j=0}^{\tau_{n}(x)} \exp \left(S_{n} \phi \circ T^{j}(x)\right) \geq \mathrm{h}_{\mu}+\int_{X} \phi d \mu .
$$

Proof. Fix $M>0$ such that $|\phi|<M$. Using Jensen's inequality, we have

$$
\begin{aligned}
\frac{1}{n} \log \sum_{j=0}^{\tau_{n}(x)} & \exp \left(S_{n} \phi \circ T^{j}(x)\right) \\
= & \frac{1}{n} \log \left(\tau_{n}(x)+1\right)+\frac{1}{n} \log \frac{1}{\tau_{n}(x)+1} \sum_{j=0}^{\tau_{n}(x)} \exp \left(S_{n} \phi\left(T^{j}(x)\right)\right) \\
\geq & \frac{1}{n} \log \left(\tau_{n}(x)+1\right)+\frac{1}{n} \frac{1}{\tau_{n}(x)+1} \sum_{j=0}^{\tau_{n}(x)} S_{n} \phi\left(T^{j}(x)\right) .
\end{aligned}
$$

The first summand on the right-hand size tends to $\mathrm{h}_{\mu}$ as $n$ tends to $\infty$. The second one can be written as

$$
\begin{aligned}
\frac{1}{n} \frac{1}{\tau_{n}(x)+1}\left(\sum_{j=n}^{\tau_{n}(x)-n} n \phi\left(T^{j}(x)\right)\right. & +\sum_{j=0}^{n-1}(j+1) \phi\left(T^{j}(x)\right) \\
& \left.+\sum_{j=\tau_{n}(x)-n+1}^{\tau_{n}(x)}\left(\tau_{n}-j+1\right) \phi\left(T^{j}(x)\right)\right) .
\end{aligned}
$$

It is easy to see that

$$
\frac{1}{n} \frac{1}{\tau_{n}(x)+1} \sum_{j=n}^{\tau_{n}(x)-n} n \phi\left(T^{j}(x)\right)=\frac{1}{\tau_{n}(x)+1} \sum_{j=n}^{\tau_{n}(x)-n} \phi\left(T^{j}(x)\right)
$$

tends to $\int_{X} \phi d \mu$, while the remaining part of the sum can be estimated by $\frac{1}{\tau_{n}(x)+1} \cdot 2 n M$. This tends to 0 a.e. since $\tau_{n}(x)$ grows exponentially fast for almost every $x$.

Let us recall the general estimate from above (see Proposition 2.3):

$$
\limsup _{n \rightarrow \infty} \frac{1}{n} \log \sum_{j=0}^{\tau_{n}(x)} \exp \left(S_{n} \phi \circ T^{j}(x)\right) \leq \mathrm{h}_{\mu}+c_{\phi, \mu}(1) .
$$

Of course, $c_{\phi, \mu}(1) \geq \int \phi d \mu$, but usually the inequality is strict, and in general we do not get any precise formula analogous to (1.11). 


\section{References}

[Bo] R. Bowen, Equilibrium States and the Ergodic Theory of Anosov Diffeomorphisms, Lecture Notes in Math. 470, Springer, 1974.

[DDL] P. Deheuvels, L. Devroye and J. Lynch, Exact convergence rate in the limit theorems of Erdös-Renyi and Sheep, Ann. Probab. 14 (1986), 209-223.

[El] R. S. Ellis, Entropy, Large Deviations, and Statistical Mechanics, Springer, 1985.

[ER] P. Erdős and A. Renyi, On a new law of large numbers, J. Anal. Math. 22 (1970), 103-111.

[OW] D. Ornstein and B. Weiss, Entropy and data compression schemes, IEEE Trans. Inform. Theory 39 (1993), 78-83.

[To] N. Torrent, Comportement asymptotique d'une somme d'exponentielles de moyennes mobiles, C. R. Acad. Sci. Paris Sér. I Paris 325 (1997), 517-522.

Laboratoire de Topologie

B.P. 47870

21078 Dijon Cedex, France

E-mail: vmaume@u-bourgogne.fr schmittb@u-bourgogne.fr

Institute of Mathematics

Warsaw University

Banacha 2

02-097 Warszawa, Poland

E-mail: aniazd@mimuw.edu.pl
Department of Mathematics University of North Texas Denton, TX 76203-1430, U.S.A. E-mail: urbanski@unt.edu

Received 26 June 2002; in revised form 14 May 2003 